\title{
Rheological characterization of polymer-based nanocomposites with different nanoscale dispersions
}

\author{
Dong Gi Seong, Tae Jin Kang, Jae Ryoun Youn*
}

School of Materials Science and Engineering, Seoul National University, Seoul 151-744, Korea; Fax +82-2-885-9671; jaeryoun@snu.ac.kr

(Received: October 19, 2004; published: January 26, 2005)

This work has been presented at the $12^{\text {th }}$ Annual POLYCHAR World Forum on Advanced Materials, January 6-9, 2004, in Guimaraes, Portugal

\begin{abstract}
Polyamide 6 - clay nanocomposites with different nanoscale dispersions were prepared by melt compounding via twin-screw extrusion and their internal structures were characterized by X-ray diffraction (XRD) and transmission electron microscopy (TEM). The rheological behaviour of these nanocomposites in shear and extensional flow were investigated using an Advanced Rheometric Expansion System and an Elongational Melts Rheometer in connection with the analysis by XRD and TEM. Nanocomposites with fully exfoliated structure and with poorly dispersed structure showed very different rheological behaviour. In general, addition of clay increased the viscosity and the storage modulus of nanocomposites, but different rheological behaviours were observed depending upon the degree of clay dispersion in the polymer matrix. In shear flow, only the exfoliated nanocomposite showed solid-like plateau behaviour in storage modulus and strong shear-thinning behaviour in shear viscosity. In extensional flow, only fully exfoliated nanocomposites showed strain-hardening behaviour, which is caused by the interaction between nanoparticles as well as between polymer molecules and nanoparticles.
\end{abstract}

\section{Introduction}

Polymer-based nanocomposites are defined as materials that contain dispersed nanometer-size particles in a single or multi-component polymeric matrix [1]. Polymer-clay nanocomposites have become an important issue in materials research because of their mechanical and thermal properties. They usually show outstanding improvements in tensile strength and modulus [2,3], heat distortion temperature [2,4], gas and liquid permeability [5,6], biodegradability [7,8], ionic conductivity [9] etc. But these improved properties are realized only when clay particles are well dispersed in the polymer matrix and close inter-particle interactions and hydrodynamic interactions exist. Therefore, it is very important to make well-dispersed structures, intercalated or exfoliated structures, and to verify the internal structure of the nanocomposites.

Many studies characterized the internal structure of nanocomposites in terms of X-ray diffraction (XRD) [10-12] and transmission electron microscopy (TEM) [13,14]. $X R D$ results represent the intragallery region of silicate layers through the diffraction 
peaks. But no peak appears when layered silicates are completely exfoliated and TEM images are frequently used to verify the exfoliated structure of nanocomposites in addition to XRD results. Nevertheless, TEM images do not provide quantitative but only qualitative results.

Rheology is an important tool for structure characterization as well as the understanding of polymer processing. The addition of various reinforcing fillers to polymers has become very popular and common to realize the requested properties, but it also increases the complexity in rheological behaviour. Rheological properties of filled polymers are influenced by filler type, size, concentration, size distribution, surface properties, etc. For example, fillers with the plate-like shape and smaller size increase polymer viscosity more than those with sphere-like shape and larger size [15]. Nanoclay has a layered structure with stacked plates of about $1 \mathrm{~nm}$ thickness and 200 - $1000 \mathrm{~nm}$ in-plane dimension [16]. Therefore, nanocomposites with nanoclay show very complex rheological behaviour.

Rheological characterization of nanocomposites is necessary in two respects. Firstly, rheological properties under different conditions should be examined to manufacture good nanocomposite products through conventional polymer processing, such as extrusion, injection molding, fibre spinning and blow molding. Secondly, rheological characterization provides information about the internal structure of nanocomposites, because nanocomposites with different internal structures show different rheological behaviour. In other words, rheological studies can be a characterization tool for the internal structure in polymer-clay nanocomposites $[1,17,18]$. In order to evaluate the structure and quality of nanocomposites rigorously, rheological characterizations are necessary in addition to XRD and TEM analysis. In summary, understanding the rheology of nanocomposites enables one to control the internal structure of nanocomposites and to develop effective processes for various nanocomposite products.

There are two distinct simple flow conditions for fluids, i.e., shear and extensional flows. Dynamic shear flow experiments were conducted to investigate the shear flow behaviour of nanocomposite melts by many researchers [1,19-21]. Most of them observed changes of storage modulus, loss modulus, and complex viscosity with respect to frequency. In the case of storage modulus at low frequency regions, nanocomposite melts showed more solid-like plateau behaviour with increasing clay content. In the case of shear viscosity, nanocomposite melts showed shear-thinning behaviour even at low frequencies while Newtonian plateau behaviour was found for pure polymer melts. Different rheological behaviours were observed with respect to the degree of dispersion of nanoclay.

In contrast to shear flow, there are only a few reports about the extensional flow behaviour of nanocomposite melts $[14,22]$. It is necessary to understand this behaviour in order to develop proper processes for the production of nanocomposite fibres, films, bottles, and other parts. In particular, homogeneous dispersion and extended orientation of clay particles are important in extensional flow and this is the reason why it is difficult to control the extensional flow process of nanocomposites.

In this study, the rheological behaviour of polyamide 6 - clay nanocomposite melts in shear and extensional flow was investigated in connection with the analysis by XRD and TEM. It was confirmed that distinct rheological behaviours were shown in shear and extensional flow due to the difference in the internal structures of nanocomposites, i.e., the degree of dispersion of nano-sized particles. In addition, discussions on the characteristic rheological phenomena will be presented in terms of the internal structure. 


\section{Experimental part}

\section{Materials}

Polyamide 6 (PA6) supplied by Kolon was selected as matrix resin of the nanocomposites since nylon 6 is known to reach an exfoliated structure easily. Unmodified montmorillonite (MMT), Cloisite $\mathrm{Na}^{+}$, was used to prepare poorly dispersed nanocomposites and organically modified montmorillonite, Cloisite 93A (C93A) from Southern Clay Products, was used to make well dispersed or exfoliated nanocomposites [23]. The same type of nanoclay was selected to obtain reliable experimental data because the two layered silicates were the same except for the added organic modifier.

\section{Preparation of nanocomposites}

Polyamide 6 and clay were melt-compounded using a Brabender counter-rotating intermeshing twin-screw extruder at the barrel temperature of $240^{\circ} \mathrm{C}$ and a screw speed of $70 \mathrm{rpm}$. The compounded melts were quenched by water and pelletized. Various samples for the analysis of internal structure and measurement of rheological properties were compression molded using a hot press (WABASH 25-1212-2TMB) at $240^{\circ} \mathrm{C}$.

\section{Characterization of the structure}

Small-angle X-ray scattering by a Rigaku Max-3 Cg X-ray diffractometer with $\mathrm{Cu}-\mathrm{K}_{\alpha}$ radiation $(\lambda=0.154 \mathrm{~nm})$ was used to determine the interlayer spacing of clays for intercalated nanocomposites. Wide-angle X-ray diffraction (M18XHF-SRA) was used to identify changes in the crystalline structure of polyamide 6 . Transmission electron microscopy (JEM-2000EX, $200 \mathrm{kV}$ ) was used to observe the dispersion state of clays in nanocomposites. TEM specimens were microtomed to an ultra-thin section with a thickness of about $80 \mathrm{~nm}$ and coated with carbon for 7 min to prevent specimens from degradation by the irradiation of electrons.

\section{Rheological characterization}

Small-amplitude oscillatory shear measurement was performed with an Advanced Rheometric Expansion System (ARES) and a Rheometric Mechanical Spectrometer (RMS) to investigate the linear viscoelastic response of nanocomposite melts in dynamic shear flow. Parallel-plate configuration with a diameter of $25 \mathrm{~mm}$ was used and the temperature was controlled at $240^{\circ} \mathrm{C}$. Maximum strain was fixed to $5 \%$, which was in the linear viscoelastic region and a frequency ranging from 0.1 to 100 $\mathrm{s}^{-1}$ was applied to the melt-state sample. Uniaxial extensional viscosity was measured with an Elongational Melts Rheometer (RME) at constant extension rate. Dimensions of the sample were $55 \times 7 \times 1.5 \mathrm{~mm}^{3}$ and the extension rate was in the range of 0.0001 and $1 \mathrm{~s}^{-1}$. The molten sample was elongated to a strain of $700 \%$ at $240^{\circ} \mathrm{C}$ and the transient extensional viscosity was obtained by measuring the applied stress.

\section{Results and discussion}

Rheology is considered as a good measure of clay dispersion and of the mechanical properties of polymer nanocomposites. In other words, rheological behaviour gives 
information on the internal structure of nanocomposites and the rheological properties should be controlled in order to make industrial nanocomposite products with superior performance. In this study, two nanocomposites with different degrees of dispersion were prepared from the same polyamide 6 and two different clays, MMT and Cloisite 93A. While polyamide 6/MMT nanocomposites had relatively poor dispersion, polyamide 6/Cloisite 93A nanocomposites showed good dispersion and a fully exfoliated structure. MMT is hydrophilic in nature and not compatible with polyamide 6 but Cloisite 93A is a natural MMT modified with a ternary ammonium salt [23], which is able to improve the compatibility with polyamide 6 and the interfacial bonding between layered silicate and polymer chain. Also, exchanged organic cations expand the interlayer spacing, which makes the polymer molecular chains penetrate easily into the interlayer regions. The rheological behaviour in shear and extensional flows was investigated in connection with the internal structure of the two different nanocomposites.

\section{Well-dispersed nanocomposites}

SAXS and TEM were used to characterize the structure of nanocomposites on the nanometer scale. The interlayer distance between adjacent silicate layers was determined from the diffraction peak in the SAXS result through Bragg's equation. Fig. 1 shows the diffraction peak of pristine organoclay, Cloisite 93A, and nanocomposites containing $1,3,5,7$ wt.- $\%$ of the organoclay. There is a diffraction peak at $3.5^{\circ}$ for pristine organoclay, which corresponds to an interlayer distance of $2.36 \mathrm{~nm}$ between the adjacent silicate layers. This diffraction peak disappears for all four nanocomposites, which means that the layered structure of clay was fully broken and an exfoliated structure was obtained. A clay content up to $7 \mathrm{wt} .-\%$ did not significantly influence the degree of dispersion.

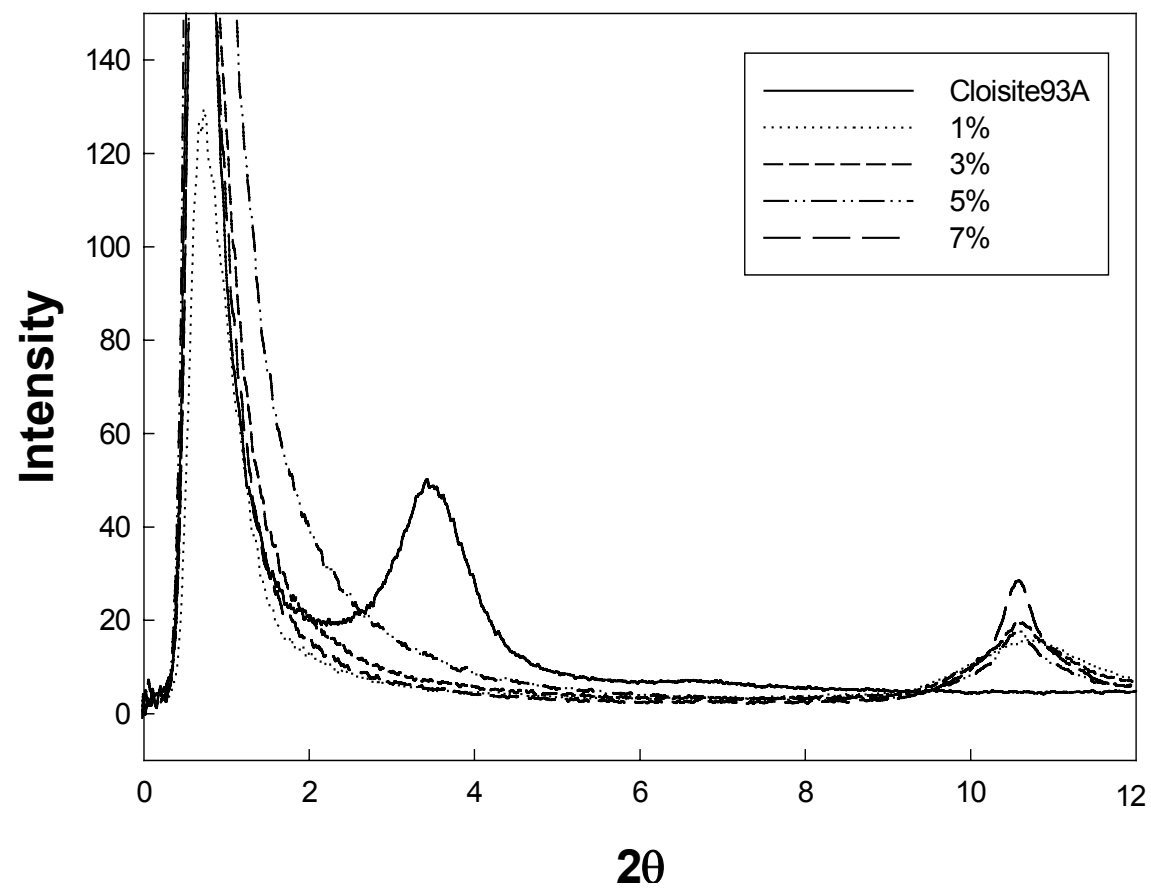

Fig. 1. Small-angle X-ray scattering peaks of Cloisite 93A and nanocomposites containing $1,3,5,7$ wt.- $\%$ clay 
This can be confirmed through TEM pictures shown in Fig. 2. Exfoliated clay layers are homogeneously dispersed throughout the polymer matrix, which is the ideal structure of nanocomposites such that hydrodynamic interaction between clay layers and macromolecular chains and particle-particle interaction of clay can be maximized.

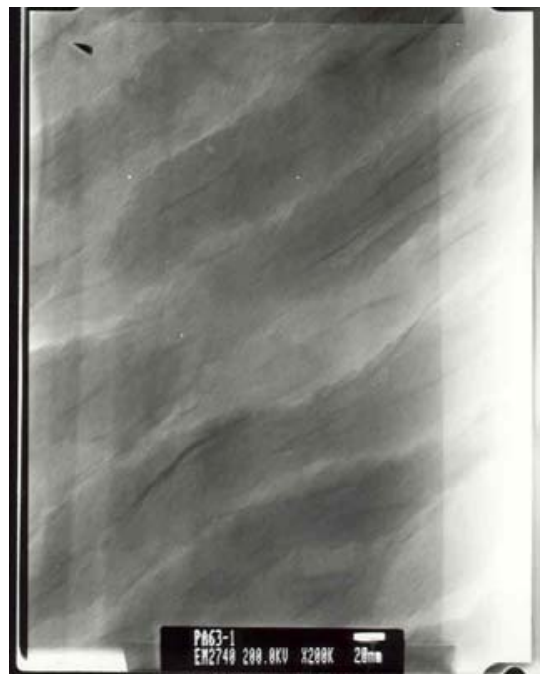

(a)

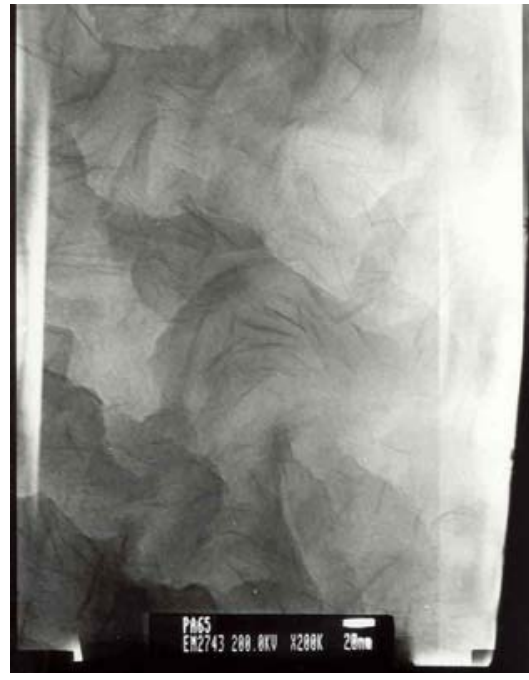

(b)

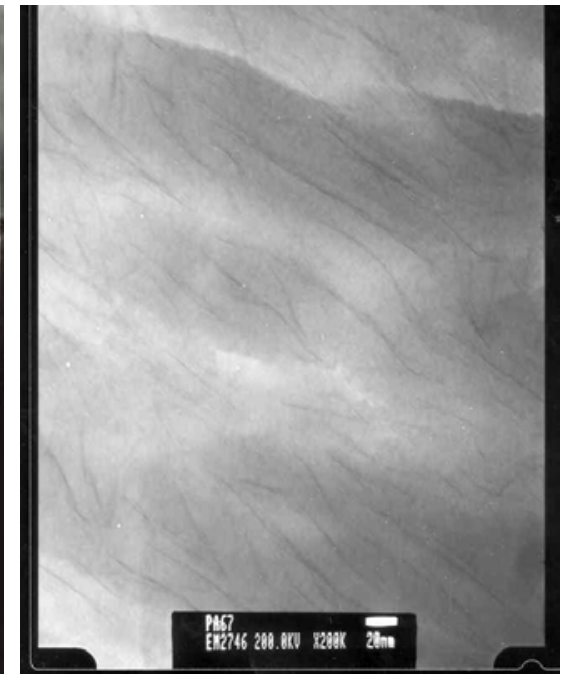

(c)

Fig. 2. Transmission electron microscope pictures of well dispersed nanocomposites (×200 000). (a) PA6/C93A (3 wt.-\%), (b) PA6/C93A (5 wt.-\%), (c) PA6/C93A (7 wt.-\%)

The rheological behaviour of the nanocomposite was investigated using dynamic shear flow and extensional flow at constant extension rates. The storage modulus of nanocomposites from Cloisite 93A is shown in Fig. 3 with respect to the clay content. Storage modulus is increased and solid-like plateau behaviour is observed at low frequencies. The solid-like plateau behaviour becomes distinct as the clay content is increased. As the amount of well-dispersed clay is increased, a network structure will be formed due to the close interaction between clay particles as well as hydrodynamic interaction between clay particles and polymer molecules. Shear viscosity, storage and loss moduli are shown in Fig. 4. Storage modulus is increased at higher rate than the loss modulus as the clay content is increased. In particular, storage modulus at low frequencies is increased remarkably, which is due to an elasticitydominant response at long time scales. In other words, the effect of clay particles on the rheological behaviour at long time scale (low frequency) is dominant. But a relatively small increase is observed at short time scale (high frequency), which indicates that the effect of clay particles on the rheological behaviour is weak and the segmental motion of polymer molecular chains is dominant at high frequencies.

Shear viscosity of the pure polymer and of nanocomposites containing Cloisite $93 \mathrm{~A}$ is presented in Fig. 5 as a function of clay content. While the pure polyamide 6 shows Newtonian plateau behaviour at low frequencies, nanocomposites show strong shear-thinning behaviour with increasing clay content. The reason is regarded as follows. While inter-particle interaction between adjacent clay particles as well as hydrodynamic interaction between clay particle and polymer molecular chain are important at higher clay content in which the distance between particles is small, only the latter is dominant at lower clay content. 


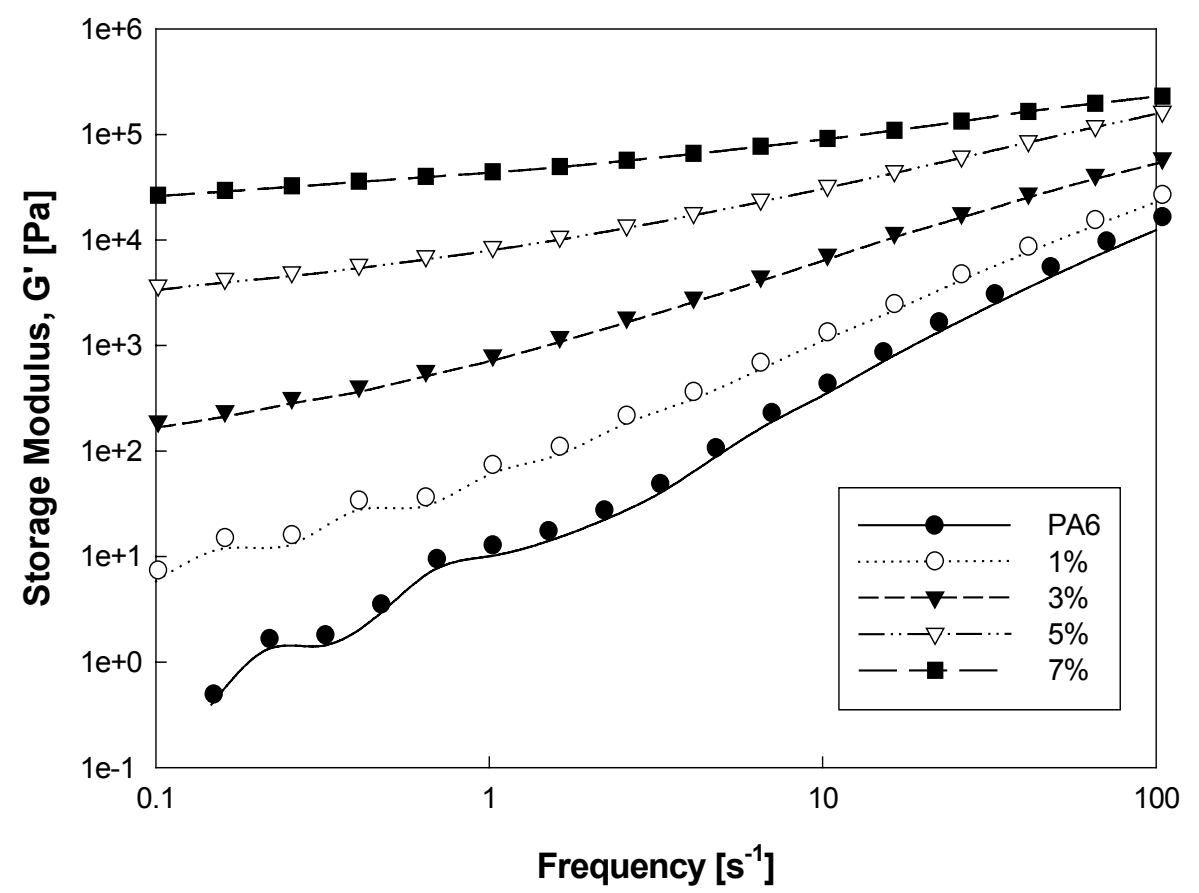

Fig. 3. Storage modulus of pure polyamide 6 and PA6/C93A nanocomposites containing $1,3,5,7$ wt.- $\%$ clay

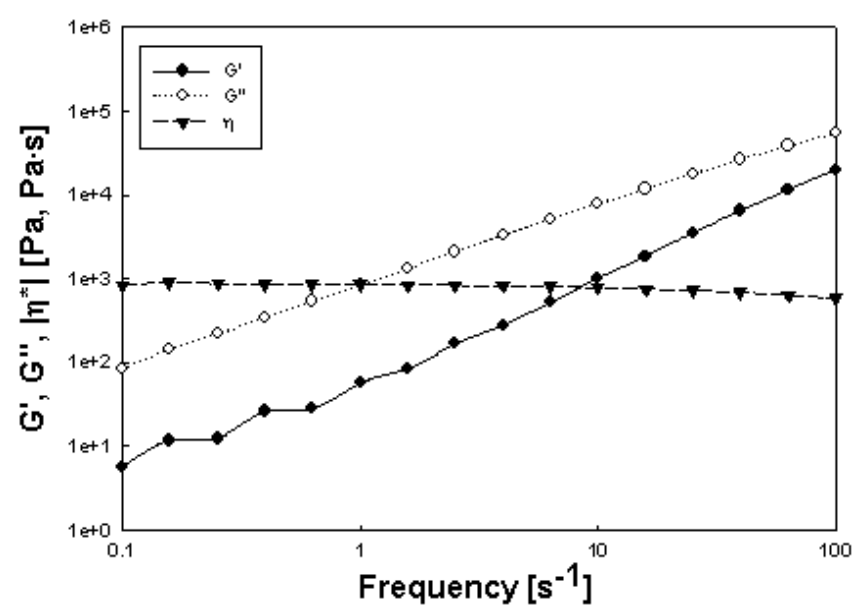

(a)

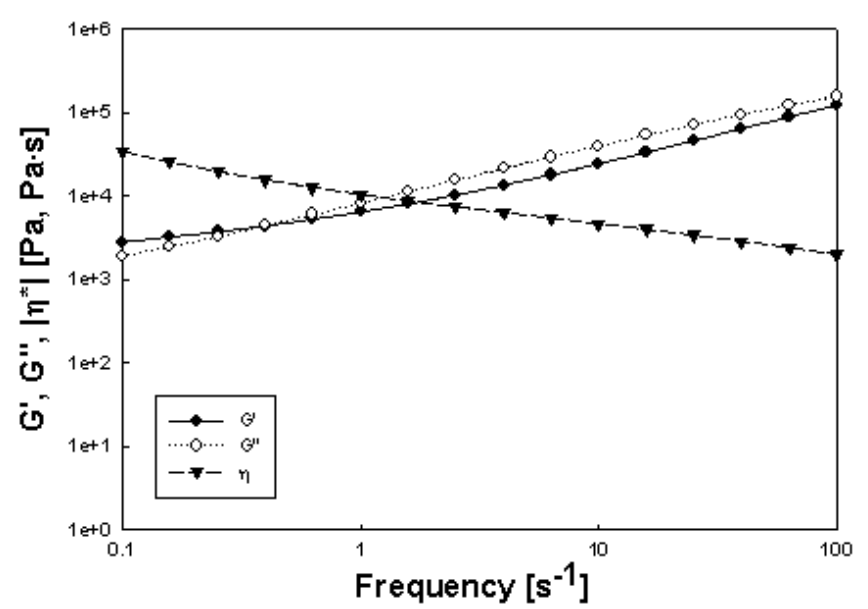

(c)

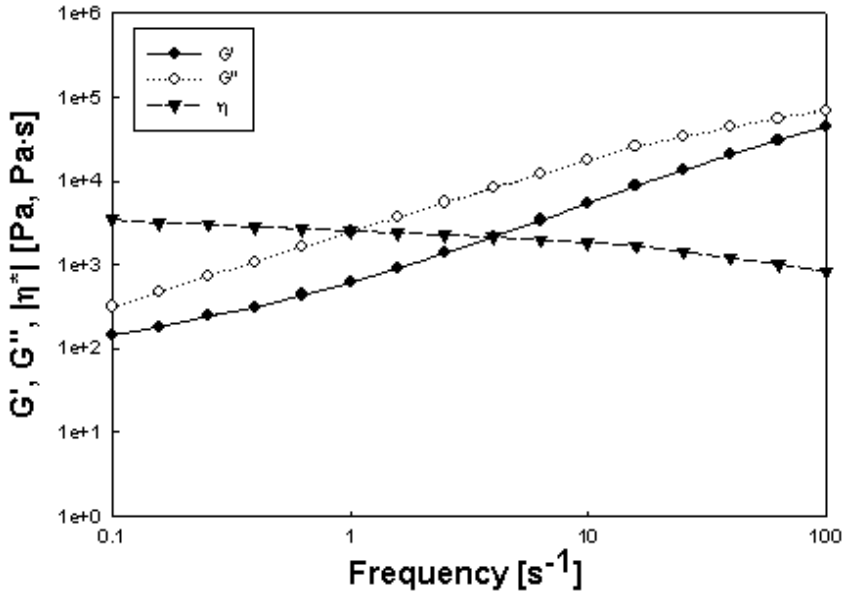

(b)

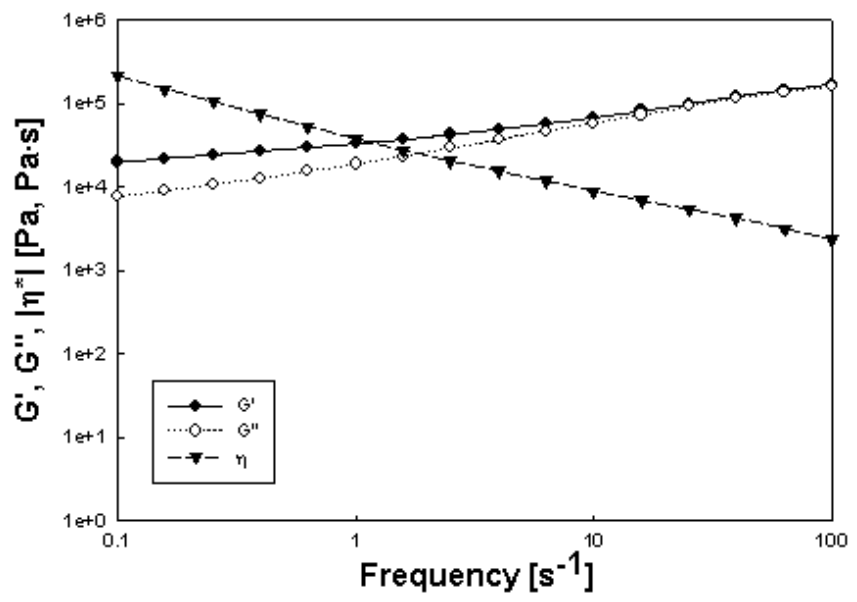

(d)

Fig. 4. Storage modulus, loss modulus, and shear viscosity of PA6/C93A nanocomposites; (a) 1 wt.-\%, (b) 3 wt.-\%, (c) 5 wt.-\%, (d) 7 wt.- $\%$ 
Extensional viscosity is also increased with respect to clay content as shown in Fig. 6 . The extensional flow behaviour of pure polyamide 6 is different from that of its nanocomposite especially in nonlinear viscoelastic flow. No strain-hardening behaviour exists in pure polyamide 6 as shown in Fig. 7 . When polymer molecules are oriented in the flow direction under uniaxial elongational flow, the linear structured polyamide 6 does not produce any additional stress that hinders the flow.

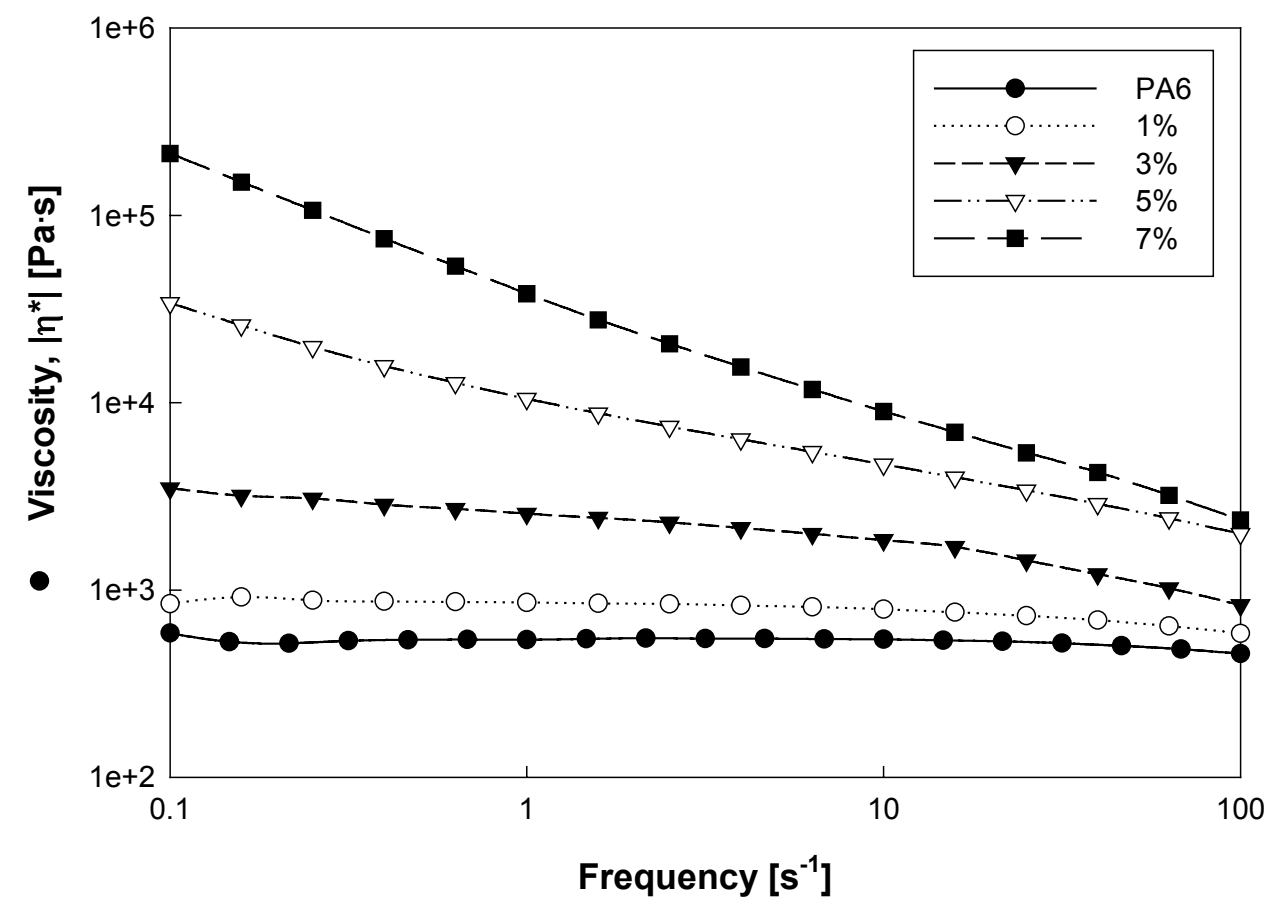

Fig. 5. Shear viscosity of pure polyamide 6 and PA6/C93A nanocomposites containing $1,3,5,7$ wt.- $\%$ clay

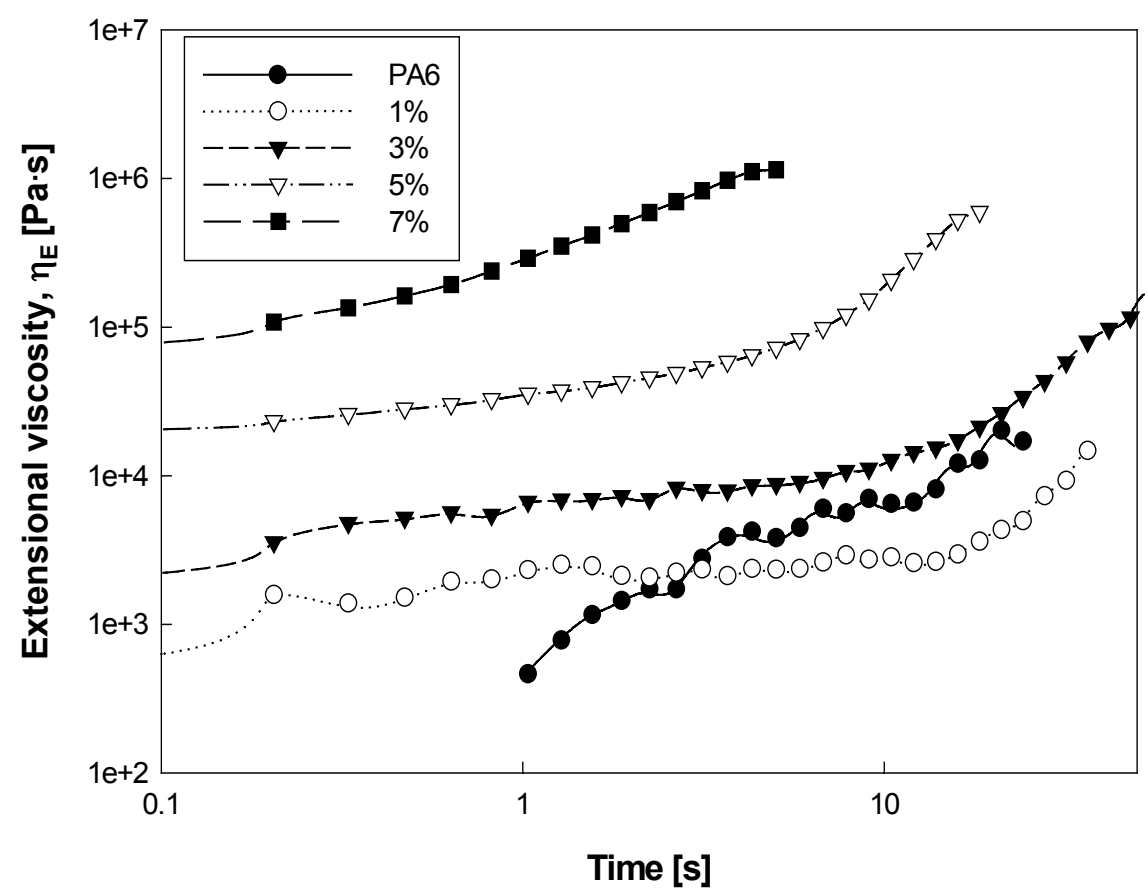

Fig. 6. Extensional viscosity of pure polyamide 6 and PA6/C93A nanocomposites containing $1,3,5,7$ wt.- $\%$ clay 
For exfoliated nanocomposites, there occurs strain hardening in the extensional flow. Strain hardening is considered as an important rheological phenomenon in the extensional flow of polymers with branched structure. It is known that side branches of polymer chains produce additional stress at uniaxial extensional flow, which provokes strain hardening. However, polyamide 6 used in this study is not a branched polymer but a linear one. The origin of strain hardening in the exfoliated polyamide 6/clay nanocomposites is regarded as follows. When the clay platelets are dispersed in the polymer matrix, they build up a network-like structure due to their close interaction, which is also called a house-of-cards structure by Okamoto et al. [14]. Friction between clay particle and polymer molecule and close inter-particle interactions hinder the uniaxial extensional flow of nanocomposite melts. The clay platelets act like branches of the macromolecular chain, which cause the additional stress in extensional flow. Thus it can be explained why strain-hardening behaviour is observed in nanocomposites. It should be noted that strain hardening is observed only in the well-dispersed or fully exfoliated nanocomposites.

(a)

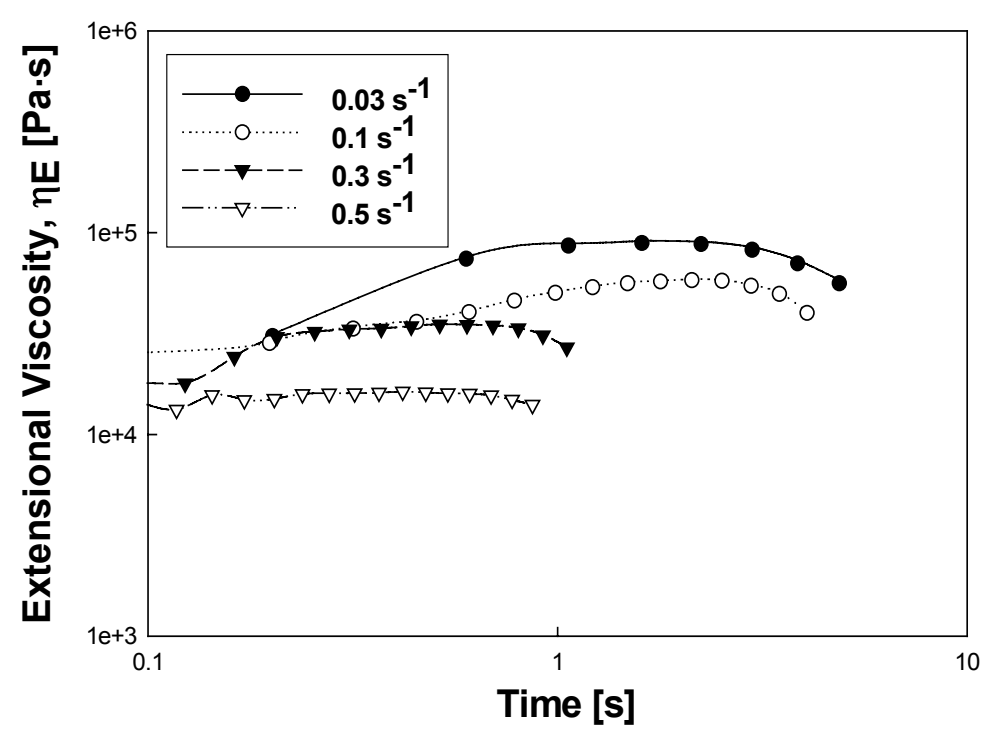

(b)

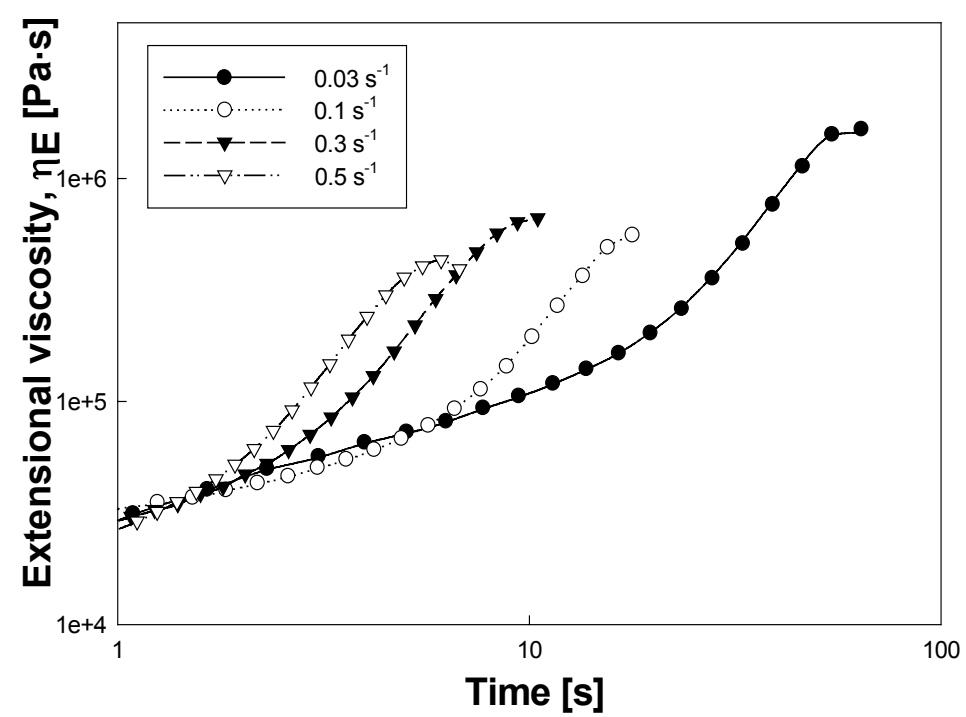

Fig. 7. Transient extensional viscosity with respect to extension rate. (a) Polyamide 6, (b) PA6/C93A (5 wt.-\%) nanocomposite 


\section{Poorly dispersed nanocomposites}

Nanocomposites containing unmodified montmorillonite, Cloisite $\mathrm{Na}^{+}$, have poorly dispersed structure in comparison with the nanocomposites containing Cloisite 93A. X-ray diffraction peaks of the unmodified clay, Cloisite $\mathrm{Na}^{+}$, and nanocomposites containing $1,3,5,7$ wt.-\% clay are shown in Fig. 8. There is a diffraction peak at $9.2^{\circ}$ corresponding to the interlayer distance of $1.17 \mathrm{~nm}$ and the peak is not observable in nanocomposites. Since there are few regular layered silicates in this nanocomposite, diffraction peaks almost disappear. This does not mean that an exfoliated structure is achieved. Instead, the nanocomposites have a disordered intercalated structure, which can be confirmed through TEM pictures as shown in Fig. 9. Nanoclay with layered structure is partially intercalated disorderly and aggregated clay exists throughout the polymer matrix. Hydrodynamic forces between clay particles and polymer chain and the particle-particle interactions of clay are relatively weak due to the clay aggregates.

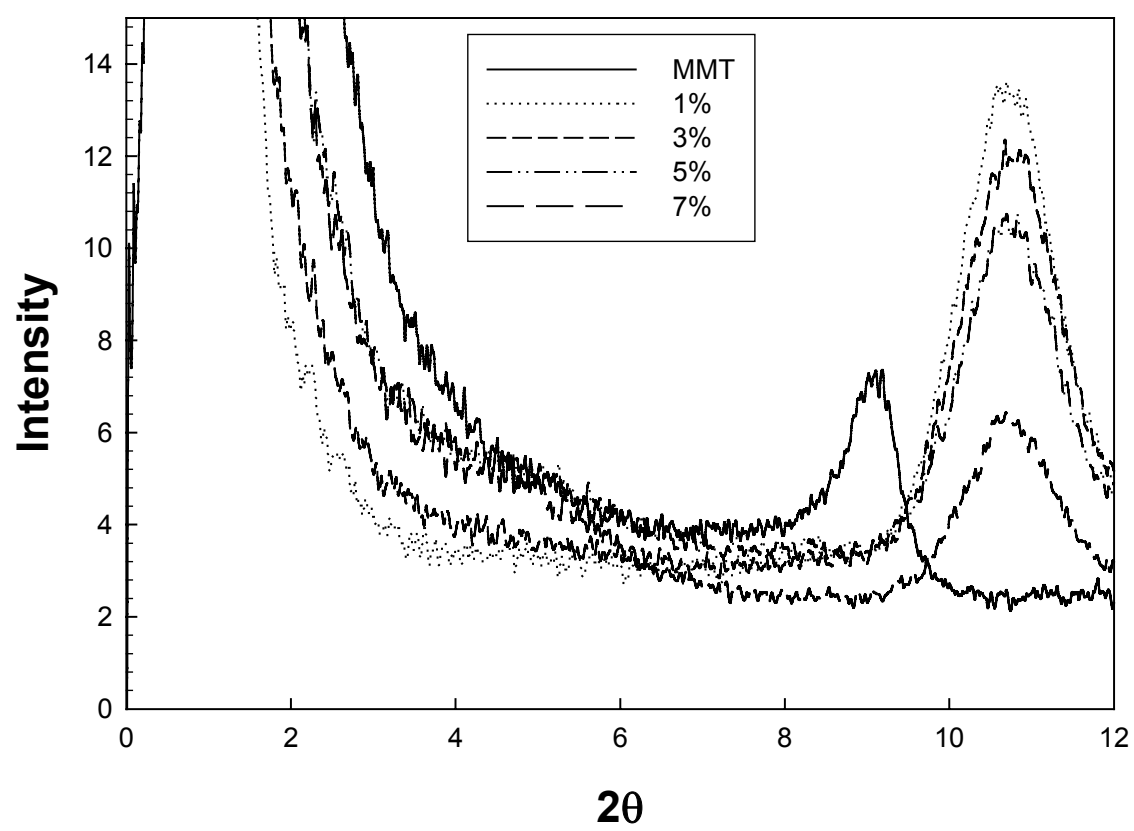

Fig. 8. Small-angle X-ray scattering peaks of the unmodified clay, Cloisite $\mathrm{Na}^{+}$, and of nanocomposites containing $1,3,5,7$ wt.- $\%$ clay

The rheological behaviour of poorly dispersed nanocomposites containing unmodified clay was different from that of the well dispersed or exfoliated ones. The storage modulus of the nanocomposite containing MMT is plotted with respect to clay content in Fig. 10. There is a slight increase of storage modulus with increasing clay content and only weak solid-like plateau behaviour is observed at low frequencies. There is no crossover behaviour at low frequency because the increase of the storage modulus is not much steeper than the increase of the loss modulus as shown in Fig. 11. There is a slight increase of shear viscosity with respect to the clay content and no shear-thinning behaviour is observed as shown in Fig. 12. These phenomena are caused by the poor dispersion of clay in the polymer matrix. The interaction between clay particles and the hydrodynamic interaction between clay particles and polymer molecules are too weak to form a network structure. Also the existence of clay aggregations, which causes the heterogeneous dispersion, interferes with the efficient dissipation of stress throughout the whole nanocomposite system. 
(a)
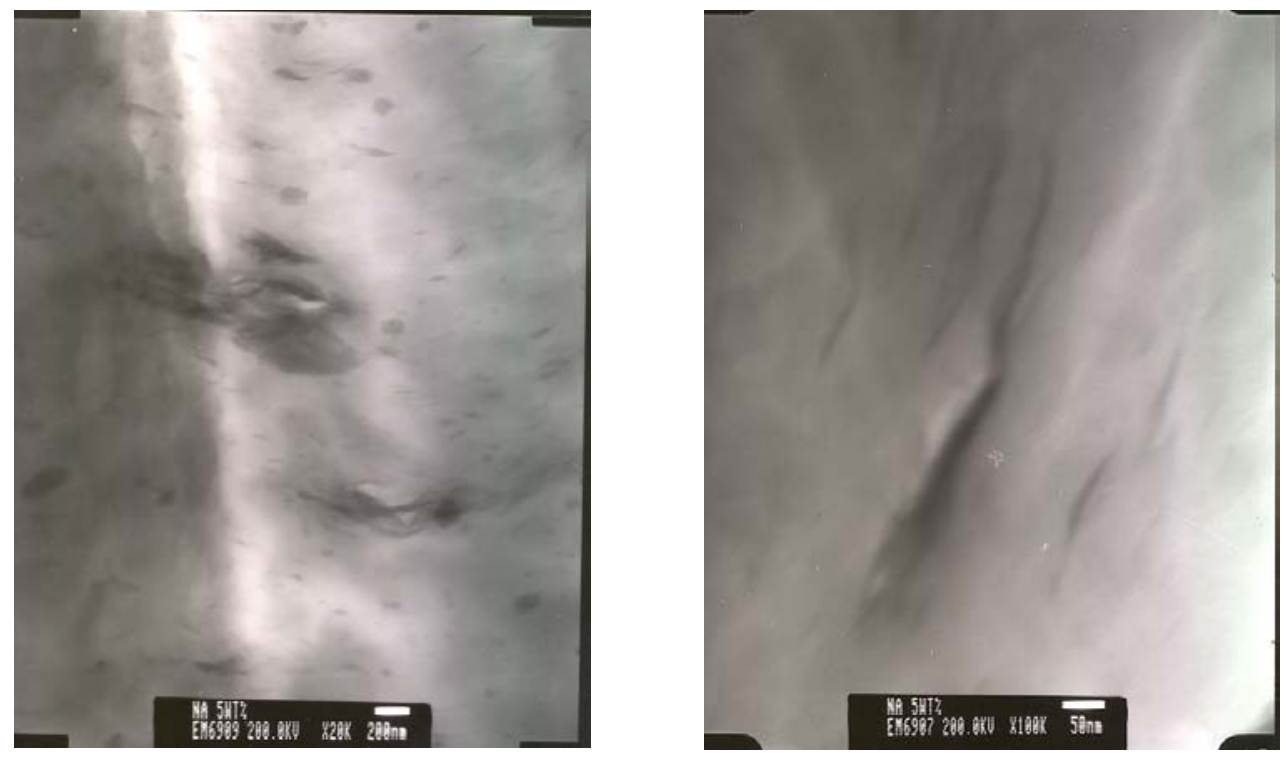

(b)

Fig. 9. TEM pictures of PA6/MMT nanocomposite containing 5 wt.- $\%$ clay. (a) magn. $\times 20000$, (b) $\times 100000$

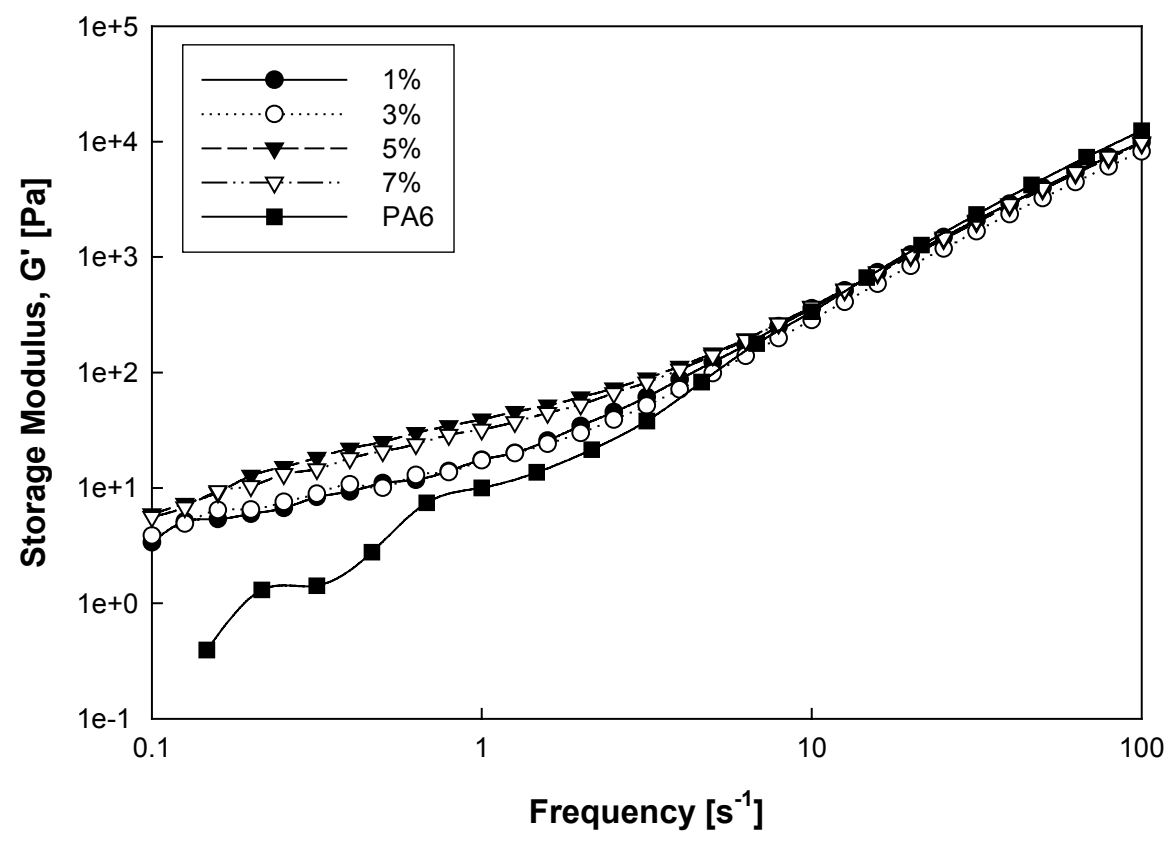

Fig. 10. Storage modulus of pure polyamide 6 and PA6/MMT nanocomposites containing $1,3,5,7$ wt.-\% clay

In extensional flow, there is no remarkable increase in extensional viscosity and strain hardening is not observed in the poorly dispersed nanocomposites as shown in Fig. 13 and Fig. 14. As clay platelets are not dispersed homogeneously in the polymer matrix, a network-like structure cannot be formed. Clay platelets with weak interaction between each other cannot produce additional stress that hinders the flow. As a result, strain hardening is hardly observed. Clay agglomerates that are observed in the polymer matrix interfere with the orientation of macromolecular chains and act as defects, which can initiate melt fracture. 


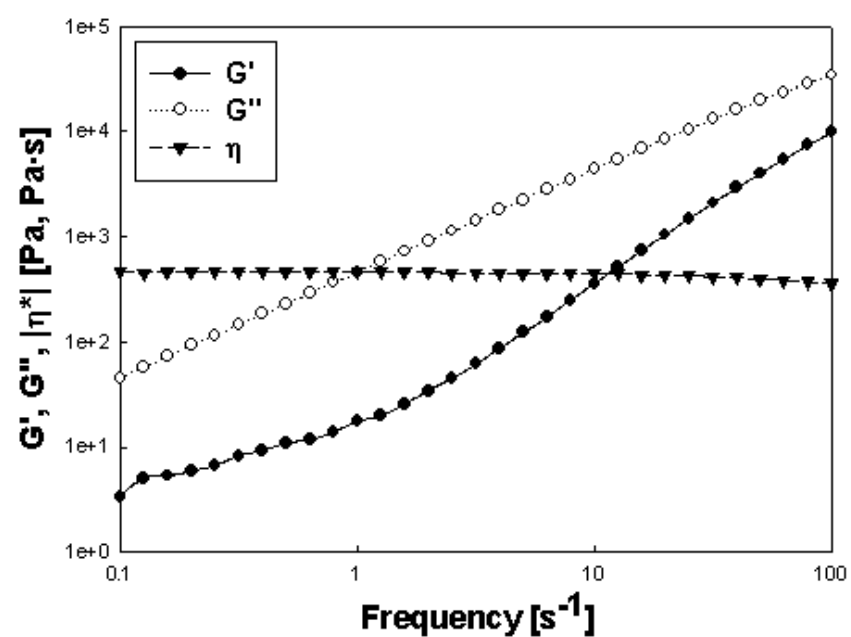

(a)

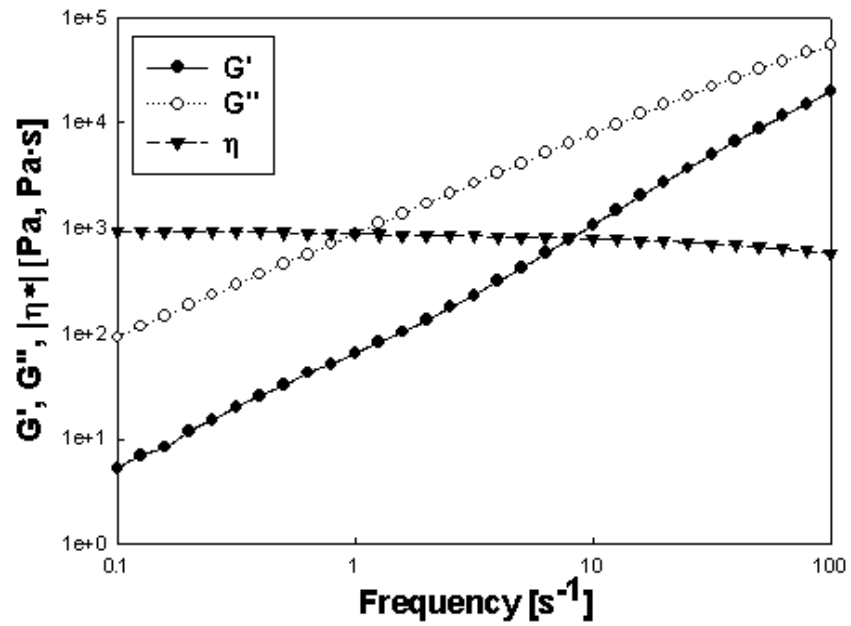

(c)

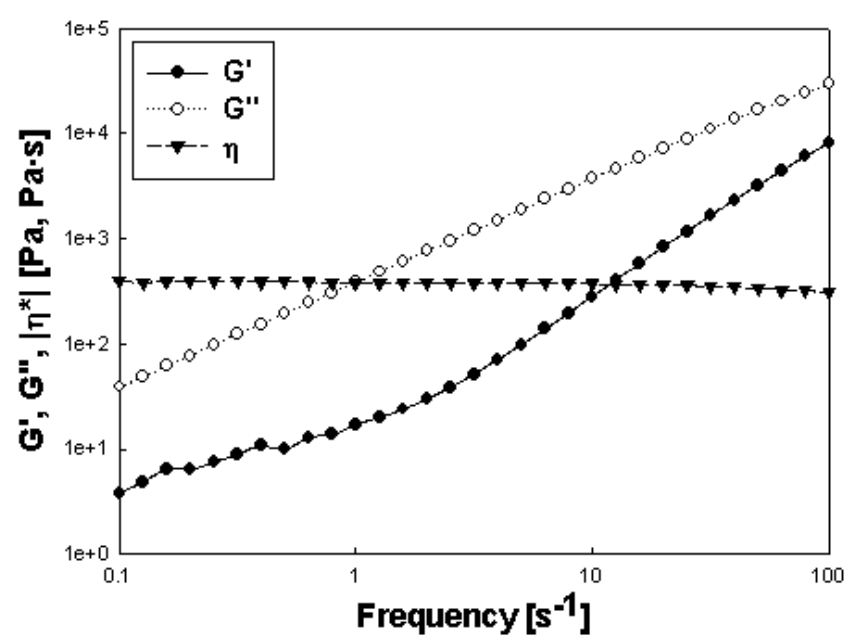

(b)

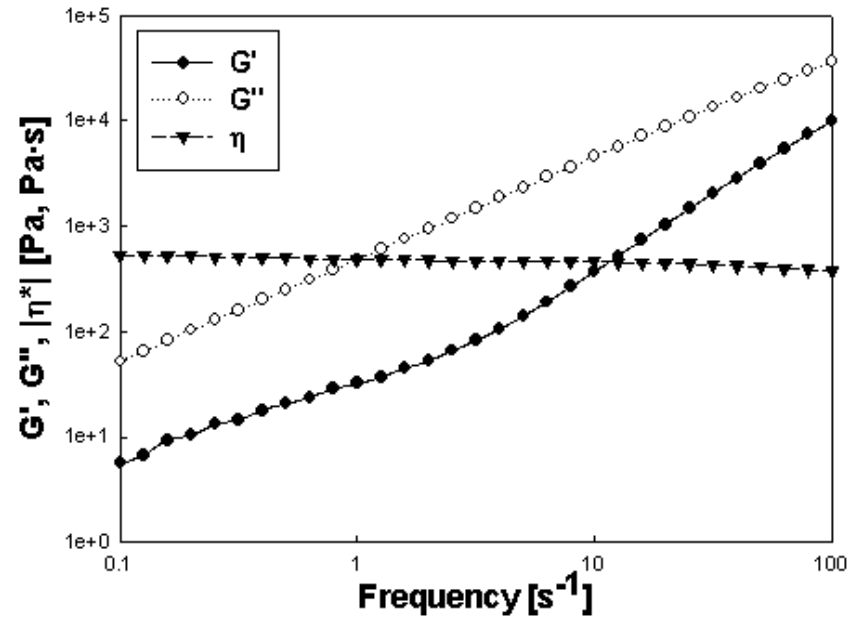

(d)

Fig. 11. Storage modulus, loss modulus, and shear viscosity of PA6/MMT nanocomposites. (a) 1 wt.-\%, (b) 3 wt.-\%, (c) 5 wt.- $\%$, (d) 7 wt.- $\%$

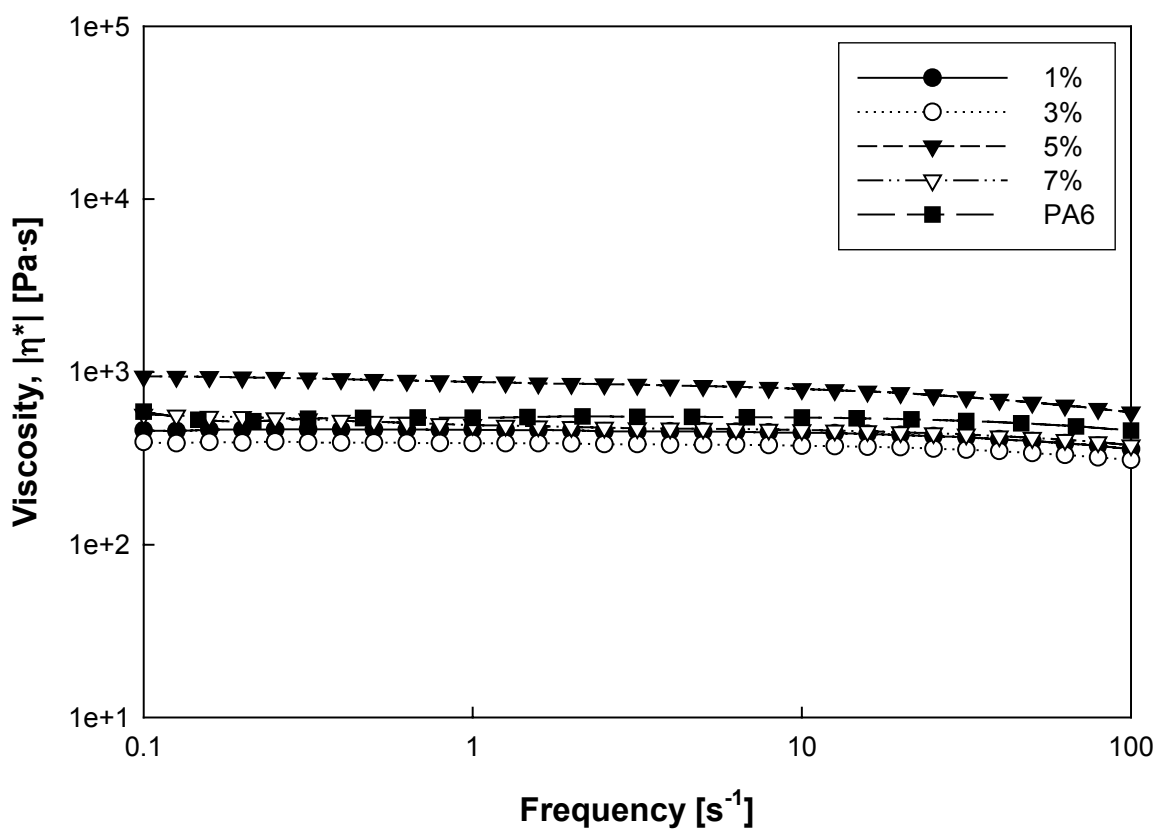

Fig. 12. Shear viscosity of pure polyamide 6 and PA6/MMT nanocomposites containing $1,3,5,7$ wt.-\% clay 


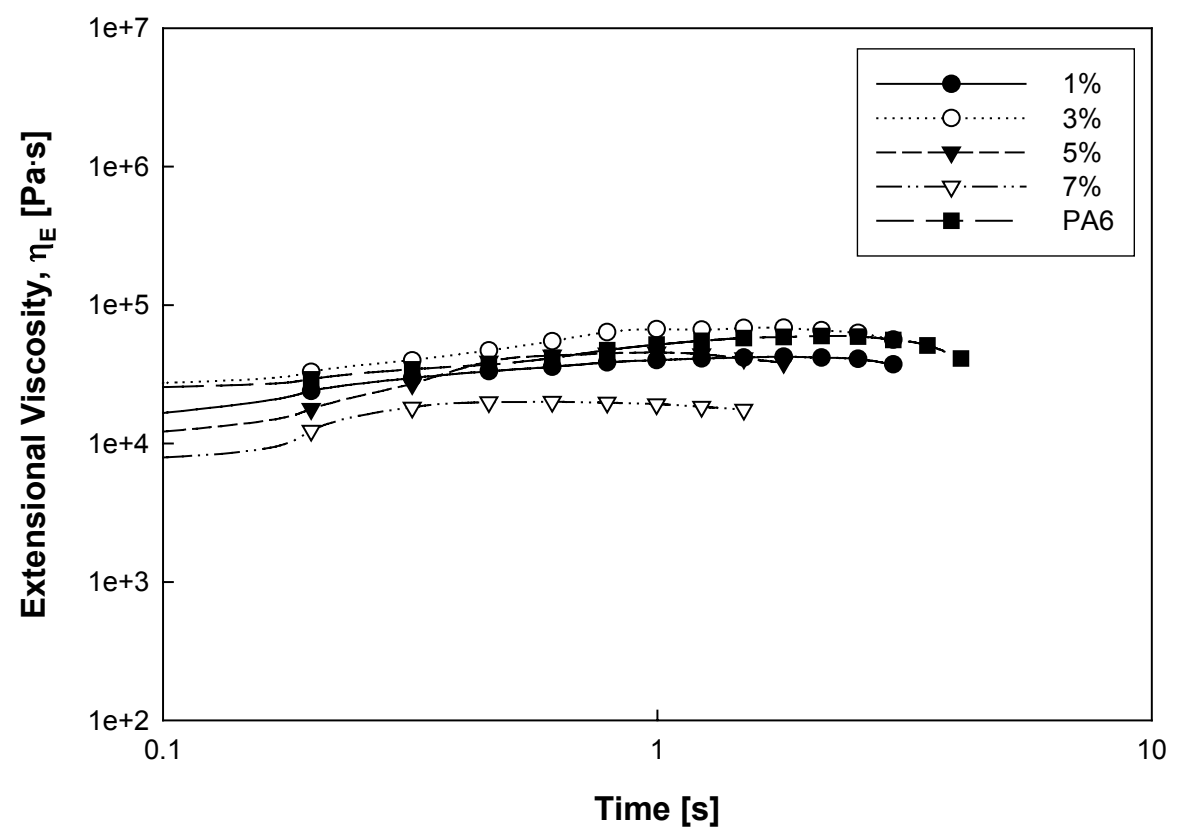

Fig. 13. Extensional viscosity of pure polyamide 6 and PA6/MMT nanocomposites containing 1, 3, 5, 7 wt.-\% clay

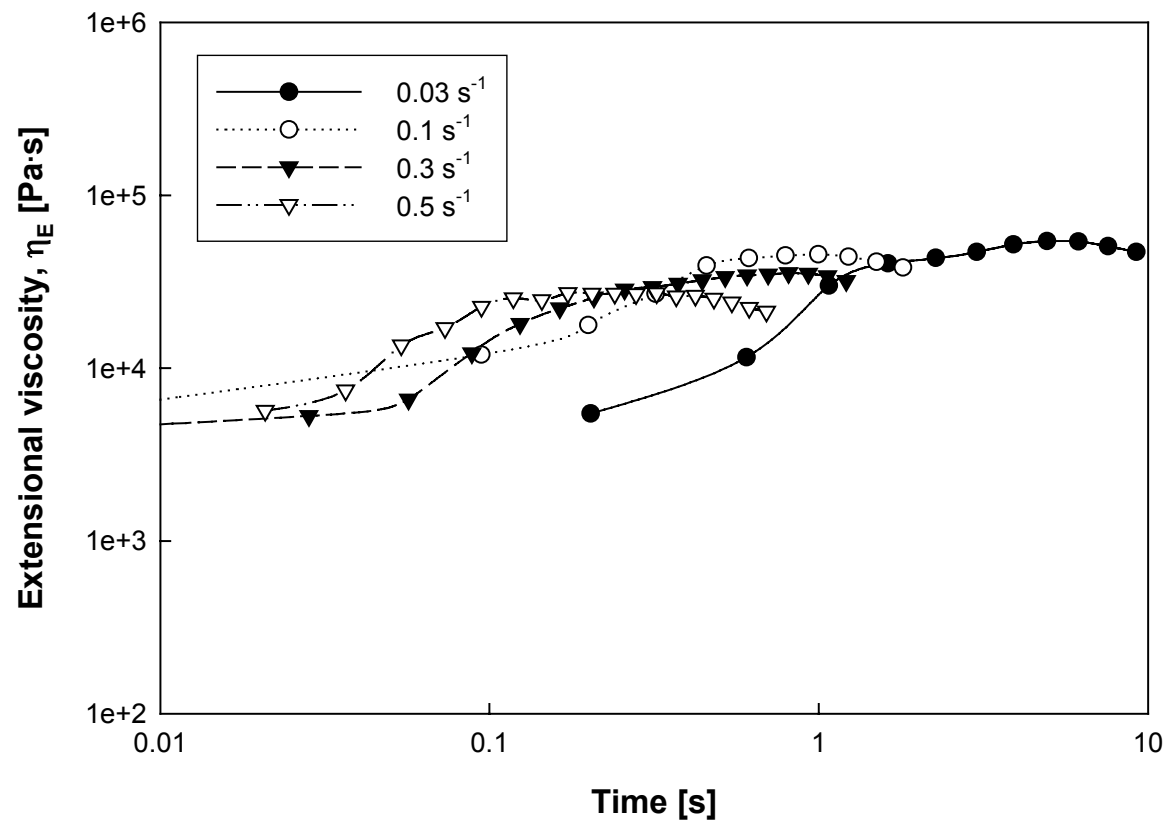

Fig. 14. Transient extensional viscosity of PA6/MMT (5 wt.-\%) nanocomposite with respect to extension rate

\section{Concluding remarks}

Rheological behaviour of nanocomposites was investigated for shear and extensional flow in connection with nanoscale dispersions. In shear flow, well dispersed nanocomposites showed large increase in storage modulus, higher viscosity, solid-like plateau behaviour, and shear-thinning behaviour. In extensional flow, strainhardening behaviour was observed only in the fully exfoliated nanocomposites. Poorly dispersed nanocomposites showed a slight increase in storage modulus and viscosity, and did not show the characteristic rheological behaviour. In exfoliated nanocomposites, the degree of solid-like behaviour in modulus and non-Newtonian 
flow behaviour were varied as a function of the clay content. While there was a Newtonian flow region at low frequency with up to $3 \mathrm{wt}$ - $\%$ of clay content, shearthinning behaviour was observed even at low frequencies with clay contents of 5 and 7 wt.-\%. In extensional flow, the frequency at which strain-hardening starts moved to a lower level as the clay content was increased. Therefore, optimal clay content should be determined on purpose and then the processing conditions such as screw speed and extension rate should be optimized from the rheological data in shear and extensional flows.

Rheology is a good tool to characterize the interactions between nanoclay and polymer molecules in nanocomposites. It is necessary to study the characteristic rheological behaviour of nanocomposites theoretically by considering the various interacting forces, e.g., the inter-particle interacting force, the friction force between particle and matrix, and hydrodynamic interactions in the dispersed system.

Acknowledgement: This study was partially supported by the Korea Science and Engineering Foundation (KOSEF) through the Applied Rheology Center (ARC) and by the Ministry of Science and Technology through the National Research Laboratory (NRL). The authors are grateful for the support.

[1] Utracki, L. A.; Lyngaae-Jørgensen, J.; Rheol. Acta. 2002, 41, 394.

[2] Kojima, Y.; Usuki, A.; Kawasumi, M; Okada, A.; Fukushima, Y.; Kurauchi, T.; Kamigaito, O.; J. Mater. Res. 1993, 8, 1185.

[3] Cho, J. W.; Paul, D. R.; Polymer 2001, 42, 1083.

[4] Kojima, Y.; Usuki, A.; Kawasumi, M.; Okata, A.; Kurauchi, A.; Kamigaito, O.; J. Polym. Sci., Chem. 1993, 31, 1755.

[5] Xu, R.; Manias, E.; Snyder, A. J.; Runt, J.; Macromolecules 2001, 34, 337.

[6] Bharadwaj, R. K.; Macromolecules 2001, 34, 1989.

[7] Tetto, J. A.; Steeves, D. M.; Welsh, E. A.; Powell, B. E.; ANTEC'99 1999.

[8] Shinha Ray, S.; Yamada, K.; Okamoto, M.; Ueda, K.; Nano Lett. 2002, 2, 1093.

[9] Vaia, R. A.; Vasudevan, S.; Krawiec, W.; Scanlon, L. G.; Giannelis, E. P.; Adv. Mater. 1995, 7, 154.

[10] Giannelis, E. P.; Adv. Mater. 1996, 8, 29.

[11] LeBaron, P. C.; Wang, Z.; Pinnavaia, T. J.; Appl. Clay Sci. 1999, 15, 11.

[12] Vaia, R. A.; Price, G.; Ruth, P. N.; Nguyen, H. T.; Lichtenhan, J.; Appl. Clay Sci. $1999,15,67$.

[13] Morgan, A. B.; Gilman, J. W.; J. Appl. Polym. Sci. 2003, 87, 1329.

[14] Okamoto, M.; Nam, P. H.; Maiti, P.; Kotaka, T.; Hasegawa, N.; Usuki, A.; Nano Lett. 2001, 1, 295.

[15] Shenoy, A. V.; "Rheology of Filled Polymer Systems", Kluwer Academic Publishers, Dordrecht 1999.

[16] Giannelis, E. P.; Krishnamoorti, R.; Manias, E.; Adv. Polym. Sci. 1999, 118, 108.

[17] Sinha Ray, S.; Okamoto, M.; Prog. Polym. Sci. 2003, 28, 1539. 
[18] Krishinamoorti, R.; Yurekli, K.; Curr. Opin. Colloid Interf. Sci. 2001, 6, 464.

[19] Hoffmann, B.; Dietrich, C.; Thomann, R.; Friedrich, C.; Mülhaupt, R; Macromol. Rapid Commun. 2000, 21, 57.

[20] Zang, Q.; Archer, L. A.; Langmuir 2002, 18, 10435.

[21] Krishinamoori, R.; Giannelis, E. P.; Macromolecules 1997, 30, 4097.

[22] Wang, K. H.; Xu, M.; Choi, Y. S.; Chung, I. J.; Polym. Bull. 2001, 46, 499.

[23] http://www.nanoclay.com, Southern Clay Products, Inc. 